\title{
A Novel Feature Extraction Method for Machine Learning Based on Surface Electromyography from Healthy Brain
}

\author{
Gongfa Li 1,3,4,", Jiahan Li ${ }^{1}$, Zhaojie Ju ${ }^{2}$, Ying Sun 1,5, Jianyi Kong 1,5 \\ 1 Key Laboratory of Metallurgical Equipment and Control Technology, Wuhan University of Science \\ and Technology, Ministry of Education, Wuhan 430080, China; ligongfa@wust.edu.cn (G.L.); \\ sunying65@wust.edu.cn (Y.S.); 15697188659@wo.com.cn (J.K.); \\ 2 School of Computing, University of Portsmouth, Portsmouth PO1 3HE, UK; zhaojie.ju@port.ac.uk. \\ (Z.J.); \\ 3 Research Center for Biomimetic Robot and Intelligent Measurement and Control, Wuhan University \\ of Science and Technology, Wuhan 430081, China; ligongfa@wust.edu.cn (G.L.); \\ 4 Institute of Precision Manufacturing, Wuhan University of Science and Technology, Wuhan 430081, \\ China; ligongfa@wust.edu.cn (G.L.); \\ 5 Hubei Key Laboratory of Mechanical Transmission and Manufacturing Engineering, Wuhan \\ University of Science and Technology, Wuhan 430080, China; 15697188659@wo.com.cn (J.K.); \\ sunying65@wust.edu.cn (Y.S.). \\ * Correspondence: ligongfa@wust.edu.cn; Tel.: +86-189-0715-9217
}

\begin{abstract}
Feature extraction is one of most important step in the control of multifunctional prosthesis based on surface electromyography (sEMG) pattern recognition. In this paper, a new sEMG feature extraction method based on muscle active region is proposed. This paper designs an experiment to classify four hand motions using different features. This experiment is used to prove that new features have better classification performance. The experimental results show that the new feature, active muscle regions (AMR), has better classification performance than other traditional features, mean absolute value (MAV), waveform length (WL), zero crossing (ZC) and slope sign changes (SSC). The average classification errors of AMR, MAV, WL, ZC and SSC are 13\%, 19\%, 26\%, 24\% and $22 \%$ respectively. The new EMG features are based on the mapping relationship between hand movements and forearm active muscle regions. This mapping relationship has been confirmed in medicine. We obtain the active muscle regions data from the original EMG signal by the new feature extraction algorithm. The results obtained from this algorithm can well represent hand motions. On the other hand, the new feature vector size is much smaller than other features. The new feature can narrow the computational cost. This prove the AMR can improve sEMG pattern recognition accuracy rate.
\end{abstract}

Keywords: sEMG; New Feature; Active Muscle Regions; Machine Learning

\section{Introduction}

Using bio-signals for human-robot interface (HRI) is an important method to achieve intuitive and friendly human-robot interface [1]. The best-known commercial application is hand prostheses, such as i-Limb [2], Cyberhand [3], Yokoi Hand [4], SJT-2 hand [5]. Many researchers have researched the control methods of prosthetic hand. In the control algorithm aspect, adaptive fuzzy sliding mode control algorithm [6], modified adaptive orthogonal matching pursuit algorithm [7] and improved local sparse representation classification algorithm [8] are put forward. Many researchers research the multi-fingered dexterous robot hand to make the hand prostheses close to the human hand. For example, optimal grasp planning of multi-fingered robotic hands [9], hand gesture recognition based on convolution neural network [10], gesture recognition based on binocular vision [11] and hand motions 
recognition based on kinect and sEMG signal fusion, mobile networks and applications [12]. The surface electromyography (sEMG) signal is a biological signal that is collected on the surface of the skin. The muscle control information of central nervous system (CNS) includes sEMG. It can be used to control electrical powered prostheses [13].

In recent years, hand motions recognition based on sEMG is an important way to control hand prosthetic devices [14], human-assisting manipulators [15,16]. Generally, the sEMG signal contains a lot of information about body movement and muscle state. [17]. Pattern recognition algorithms were introduced to discriminate the sEMG signals of different hand motions. Therefore, this method can provide human hand movement control for prosthetic hands [18]. Figure 1 shows the basic flow of sEMG pattern recognition. Distinct and repeatable patterns extracted from sEMG contribute to a successful application of pattern recognition methods. There has been an increase in both the quantity and quality of identifiable motions [19].

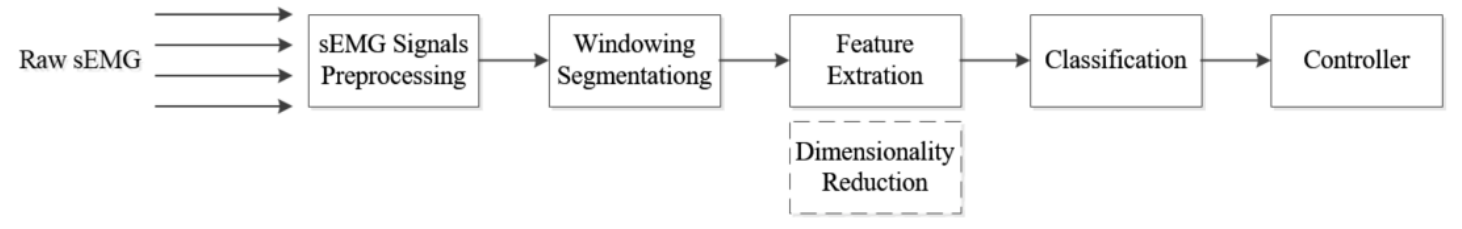

Figure 1. sEMG pattern recognition flow chart

The three main modules composing a pattern recognition method is data segmentation, feature extraction and classification [20]. Data segmentation divide sEMG data stream into segments of same window length. Feature extraction extract the most representative features of the signals to be classified. Classification classify samples into predefined categories according to their features and classification criteria. The first step mainly depends on the experience of the experimenter. As shown in Fig.1, in pattern recognition, feature extraction is the most important step to improve the recognition accuracy rate [21].

Therefore, many researchers have begun to study the features of the sEMG signals, such as time domain features [22], time-frequency features [23] and high order statistics [24]. Autoregressive model analysis is also a common method to get signal feature [25]. As a new feature, the feature DRMS, median frequency (MDF) and mean frequency (MNF) have been proposed in recent years [26,27]. However, the performances of these features were weak [28]. Some researchers began to study some new features which are not part of time domain and frequency domain, such as nonlinear properties [29] and fractal features [30,31]. In addition, multiple features are studied or evaluated to improve the hand motion classification accuracy. $[32,33]$.

This paper focus on proposing a new feature extraction method for improving sEMG classification. This new feature is based on active muscle patterns for dynamic hand motions. We prove the mapping relationship between the active muscle areas and the hand motions based on previous research. sEMG can evaluate and record the degree of muscle activity and also can quantify the contraction of the muscles [1]. This paper chooses sEMG to measure the intensity of muscle activity. Firstly, we divide the forearm skin surface and mark the number. This paper extract more active muscle regions during hand motions. We get new feature through muscle active regions.

sEMG time domain features have been proved to have good performance in hand gesture recognition. [21]. This paper compares the new feature with several traditional time domain features, such as ZC (Zero Crossing counts) [34], SSC (Slope Sign Changes) [35] and WL (Waveform Length) [36]. The experimental result shows that this new feature can improve the accuracy of different hand motions classification based on sEMG. At the same time, the data quantity of the new feature is much smaller than the traditional one. The new features can reduce the computation cost and computation time of pattern recognition methods. The new method mentioned in this paper helps to realize the friendly human-machine interaction 
between intelligent prosthetic hand and user. It also helps to realize the real-time and accurate control of prosthetic hand.

The rest of this paper is organized as follows. Section 2 describe the experimental data collection methods and feature extraction methods. Section 3 analysis experimental results. Section 4 draws the conclusion, gives the discussion of future work.

\section{Methods}

\section{1. sEMG signals collection}

Nine subjects were involved in the experiment. All of them were healthy able-bodied subjects, without any prior neuromuscular disorders (eight males and one female, aged 20-30, denoted as P1 to P9).

sEMG is usually collected by one or more electrodes were placed on the skin surface [37]. Electrode shift and electrode distribution will obviously affect the recognition accuracy of the hand motion $[38,39,40]$. Experiments use a 16-channel electrode sleeve to collect experimental data. Wet/dry electrodes can collect sEMG signals [41]. In this paper the electrodes are modified from standard disposable $\mathrm{Ag} / \mathrm{AgCl}$ ECG electrodes by gel removing means. Elastic fabrics are used to fix electrodes in advance. This method can reduce the time and position error of arranging electrodes. To obtain better skin impedance, the subjects must use alcohol to wash the forearm skin before the experiment [26]. In this paper, sEMG signal sampling frequency is set to $1 \mathrm{kHz}$, which is twice higher than the maximum frequency of sEMG signals and conforms to the nyquist sampling theorem.

Electrode sleeve is worn in the subject's right hand to collect data. Subjects use the ulna as a reference to wear the 16-channel electrode sleeve. The ulnar coincides with the midline of the connection of electrode 8 and electrode 16 and the connection of electrode 1 and electrode 9 . Sleeve wear position is shown in Figure 2.

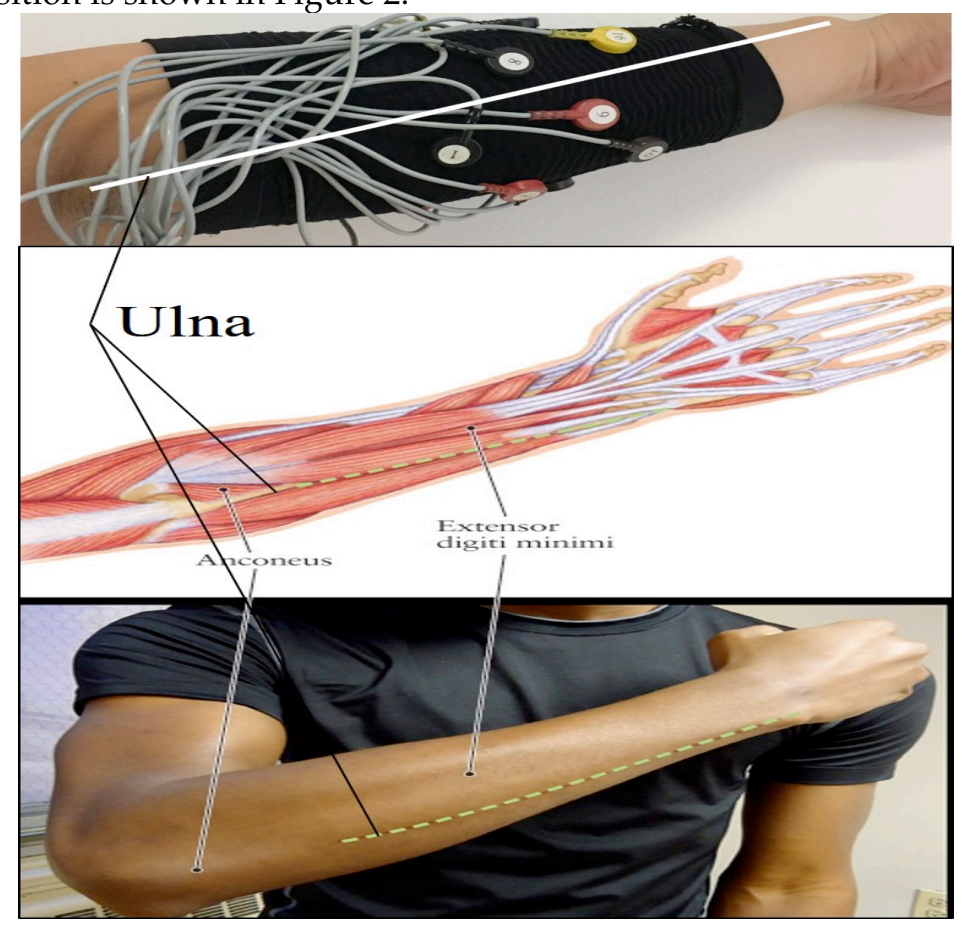

Figure 2. The reference position to wearing the 16-channel electrode sleeve

Each subject wears the electrode sleeve for about 2 minutes. This operation can make the electrode fully contact with the skin to reduce signal noise. The experiment collects the sEMG signals of 4 different hand motions of each subject. The four motions were fist firstly and then turn left wrist, turn right wrist, turn the wrist up and down the wrist. Experimental motions 
are shown in Figure 3. The subjects repeated each action 12 times. The experimenter needs to complete the hand motions within 3 seconds.

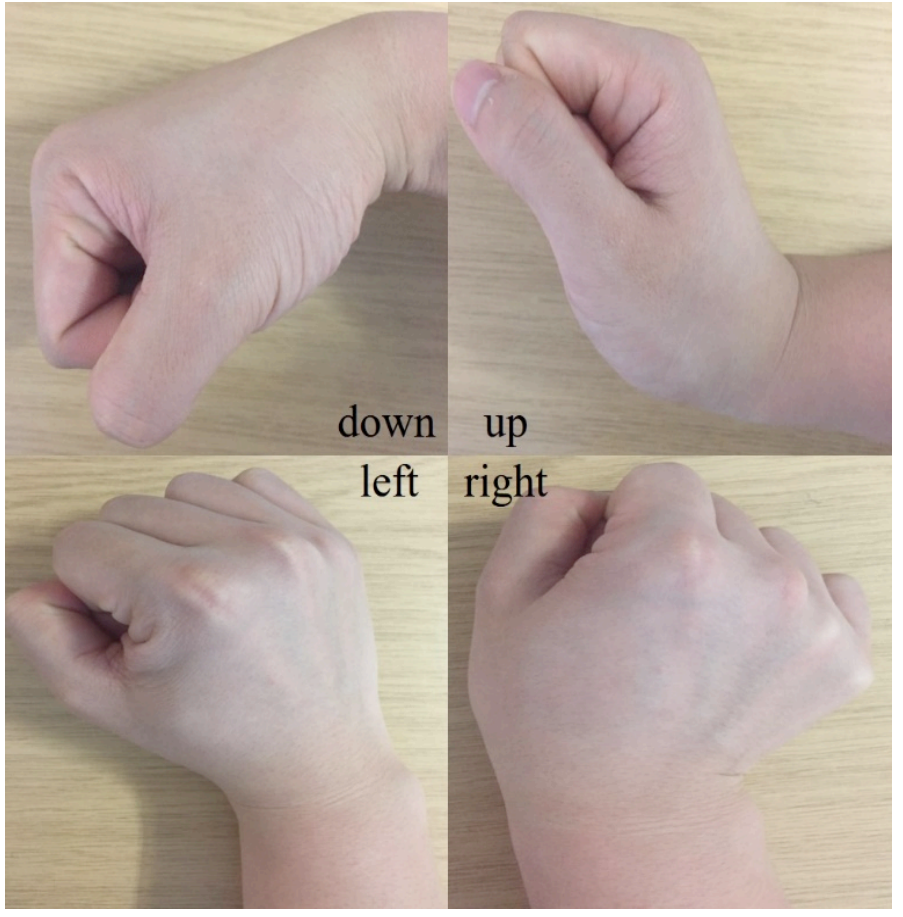

Figure 3. The experimental motions

The sEMG collector comes with a $50 \mathrm{HZ}$ comb filter and a $20 \mathrm{HZ}$ high-pass butter worth filter [42]. It could filter out most of the common noise and the dry electrode on the sleeve is uniformly distributed on the forearm [43]. The original sEMG signals are filtered skin surface voltage. The subject's experimental motions are completed in 3 seconds. Each action window contains 2 seconds of rest time and 1 second of action time. The experimental process is shown in Figure 4. The captured sEMG signal consists of four phases: The relax state of a hand, the dynamic procedure for forming a hand motion, the steady state for maintaining a hand motion and the dynamic procedure for releasing a hand motion $[44,45]$. In order to avoid the influence of redundant signals, this paper intercepts the action signal with a window size of 1 second. This paper collected 432 hand motion signals.

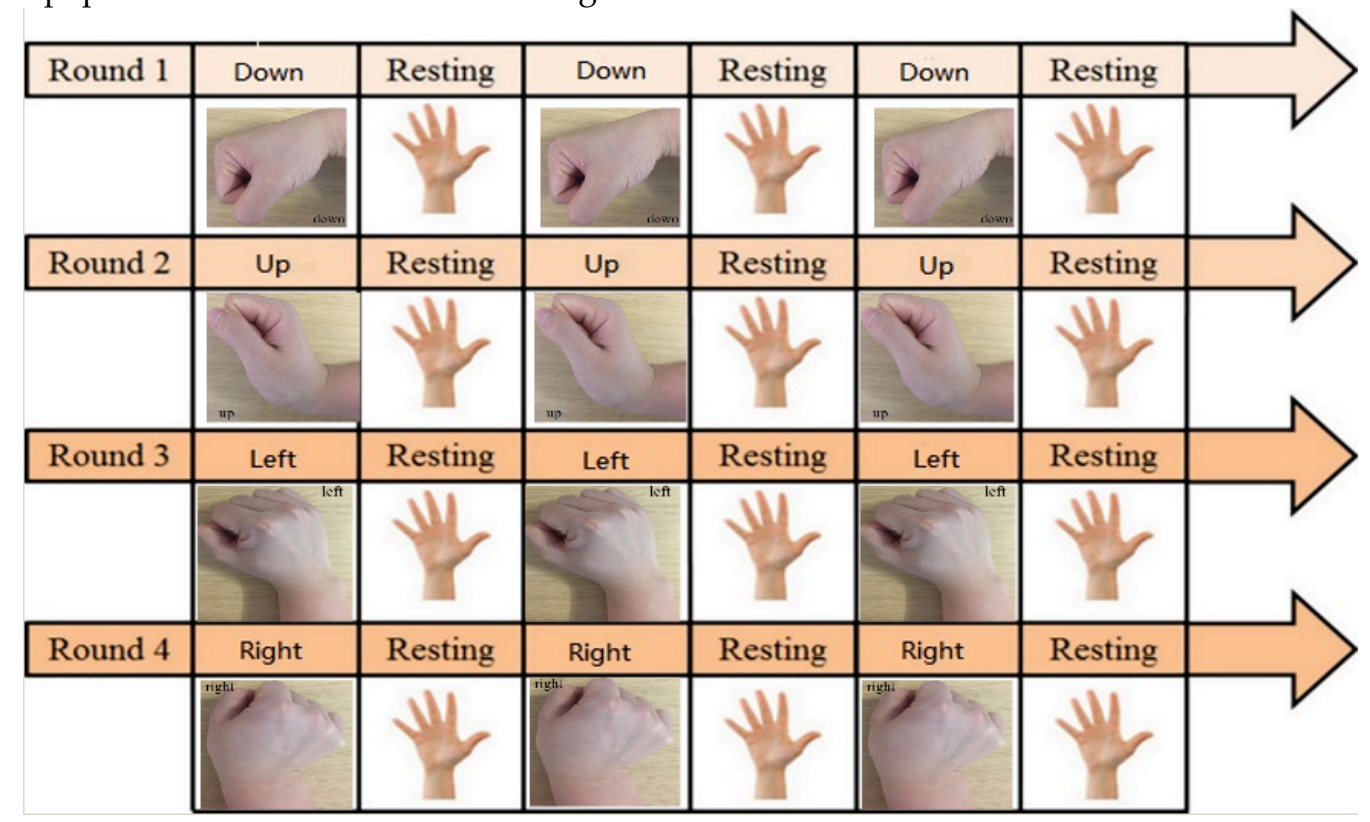

Figure 4 . The experimental process 


\subsection{Feature extraction}

The collected data with 16 channels are put into a buffer under a customized format. Once the buffer is full, a data parcel would be copied to a special buffer in the USB unit and prepared to be read by the USB host in a PC.

sEMG can evaluate and record the degree of muscle activity and also can quantify the contraction of the muscles. Different actions are done by different muscles. The contractions of the major active muscles are significantly higher than others. The degree of muscle contraction is positively correlated with the intensity of sEMG signal. Different muscles are distributed in different regions of the forearm. Subject motions and muscle active areas would show mapping relations.

This paper chooses mean absolute value (MAV) to measure the intensity of sEMG signal. Mean absolute value (MAV) is calculated for each hand motion signals. MAV is the average rectified value (ARV) and the moving average of full-wave rectified SEMG can be used to calculate it. It is defined as (1). where $\mathrm{N}$ represents the length of the signal and $\mathrm{X}_{n}$ represents the EMG signal in a segment [46].

$$
M A V=\frac{1}{N} \sum_{N=1}^{N}\left|\mathrm{x}_{n}\right|
$$

The 2-D arrangement of the experimental electrodes are shown in Figure 5, where 17 and 18 electrodes are ground wire. According to the reference position to wearing the 16-channel electrode sleeve, the positional relationship between the muscle and the electrode shown in Figure 6. the experimental positional relationship will have a small deviation.

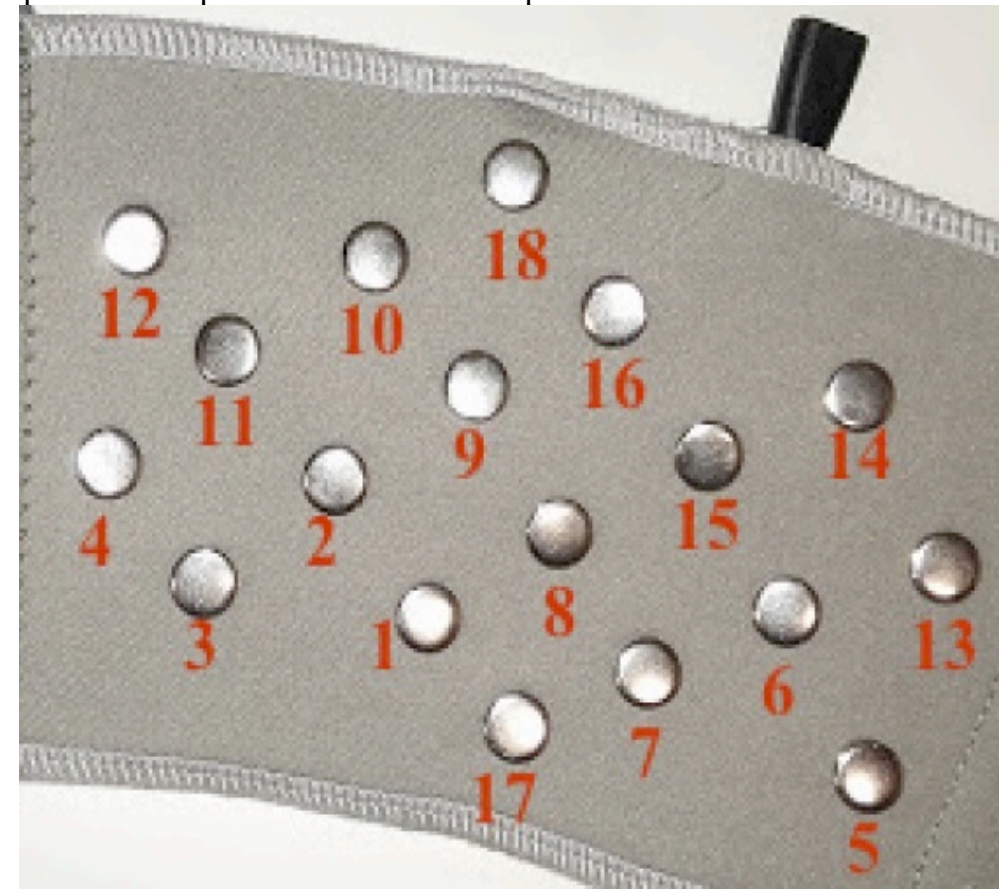

Figure 5. The 2-D arrangement of the experimental electrodes (17 and 18 electrodes are ground wire) 


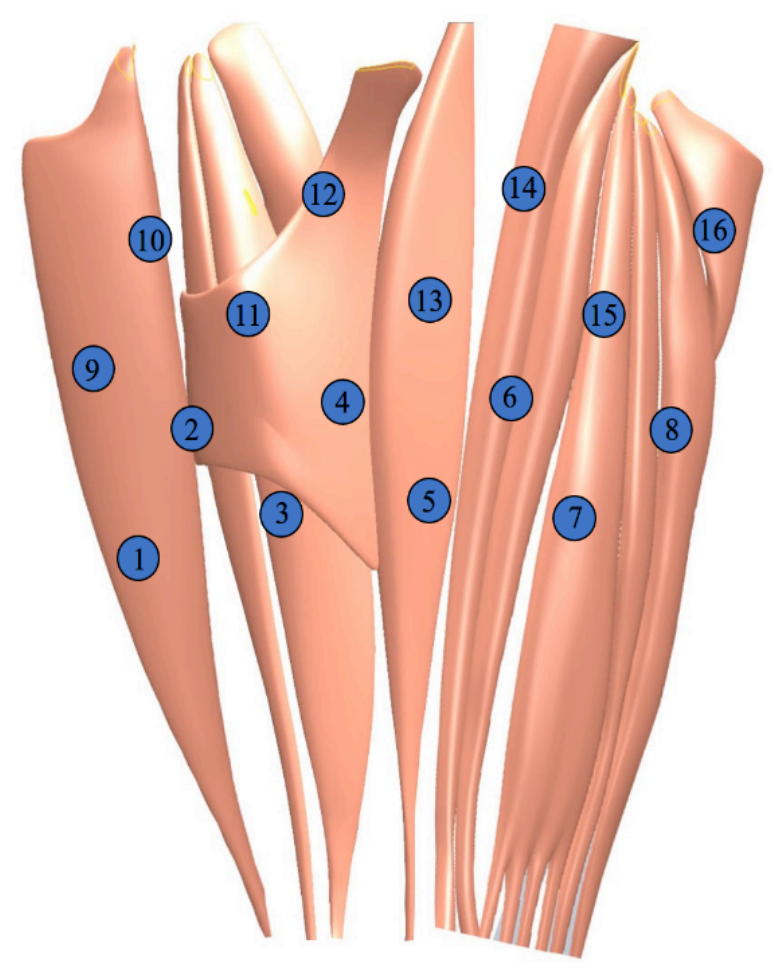

Figure 6. Positional relationship between the muscle and the electrode

According to this positional relationship and the distribution of signal intensity, this paper combines image technology to build a muscle activity visualization system. This system is based on the position relationship between the location of muscle and electrodes, and the mapping between the $\mathrm{H}$ value in HSV color space and the intensity of EMG signal. Different colors represent different intensity of muscle activity. This system can display the muscles active level in different hand motions. For example, the corresponding muscle activity of two different hand motions is shown in Figure 7.

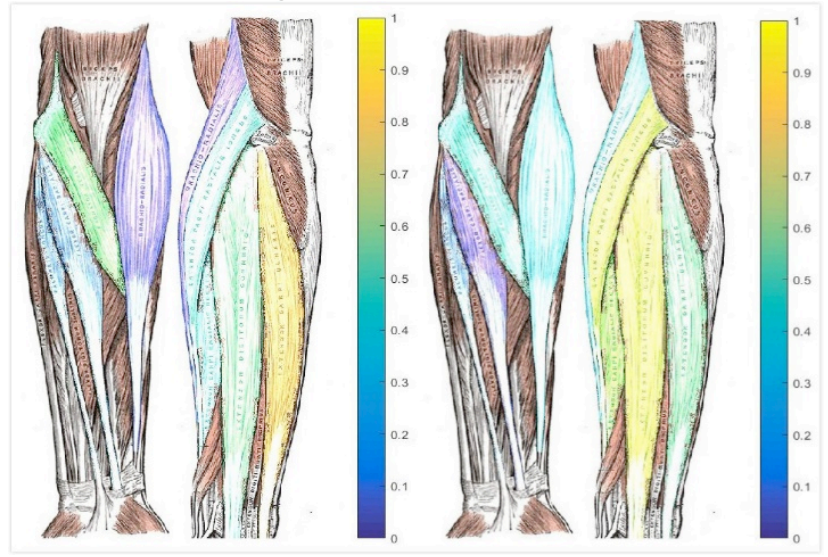

Figure 7. Muscle activity of two different hand motions (Color represents the degree of muscle activity)

According to the results of Figure 7, when the hands do different movements, different muscles show different degrees of activity. Based on the above analysis, the forearm surface is divided into 16 regions. Each region uses an electrode to measure muscle activity level. This paper uses the Mean Absolute Value (MAV) to quantify the muscle activity level. This paper equally divides the 1 s complete motion signal into 5 sections. Each section contains a $200 \mathrm{~ms}$ motion signal which has been widely accepted to signal acquisition systems $[47,48]$. This paper calculates the MAV of each channel for each signal. The calculation is a set of 16 elements. Each element is a two-dimensional coordinate. This set is shown in (2). $x$ is the number of muscle region. $y$ is the Mean Absolute Value (MAV) of the corresponding region. $M_{2}$ is a set of $y$, this set is shown in (3). $M_{3}$ and $M_{4}$ are subsets of $M_{2}$. If $M_{2}, M_{3}$ and $M_{4}$ 
satisfies the conditions in (4) and (5), the result of $M_{3}$ is output. The $M_{3}$ is shown in (6). According to $M_{3}$ and $M_{1}$, the results of $M_{5}$ can be obtained. The $M_{5}$ is shown in (7). Vector $V$ can be obtained from $M_{5}$, which is defined as (8).

$$
\begin{gathered}
M_{1}=\left\{\left(x_{1}, y_{1}\right),\left(x_{2}, y_{2}\right), \ldots \ldots .,\left(x_{16}, y_{16}\right)\right\} \\
M_{2}=\left\{y_{1}, y_{2}, \ldots \ldots, y_{16}\right\} \\
M_{3} \subseteq M_{2} ; M_{4} \subseteq M_{2} ; \\
M_{3} \cap M_{4}=\Phi ; M_{3} \cup M_{4}=M_{2} \\
\operatorname{Min}\left(M_{3}\right)>\operatorname{Max}\left(M_{4}\right) ; \operatorname{Card}\left(M_{3}\right)=4 \\
M_{3}=\left\{y_{a}, y_{b}, y_{c}, y_{d}\right\} \\
M_{5}=\left\{x_{a}, x_{b}, x_{c}, x_{d}\right\} \\
V=\left(x_{a}, x_{b}, x_{c}, x_{d}\right)
\end{gathered}
$$

The new feature is named Active Muscle Region (AMR). The window length determines how many samples will be used to generate one decision. Here we compared the window length from $50 \mathrm{~ms}$ to $400 \mathrm{~ms}$ for offline evaluation and the result is shown in Figure 8. The trend is easily observed that larger window length contributes to better recognition accuracy. However, there exists a tradeoff between delay and recognition accuracy. It has been reported that a delay of more than $300 \mathrm{~ms}$ is perceivable to prosthetic users. Based on the above reasons, sEMG features were extract by a moving window algorithm of 1000-ms time window with 200-ms increase window. This algorithm was trying to smooth sEMG signals and avoid edge effects [49]. As shown in Figure 9. A hand motion signal is calculated to get 5 sets. This paper uses the 20 elements in the set to get a vector. Therefore a $20(1 \times 20)$ dimensional feature vector for each frame of sEMG signals is obtained.

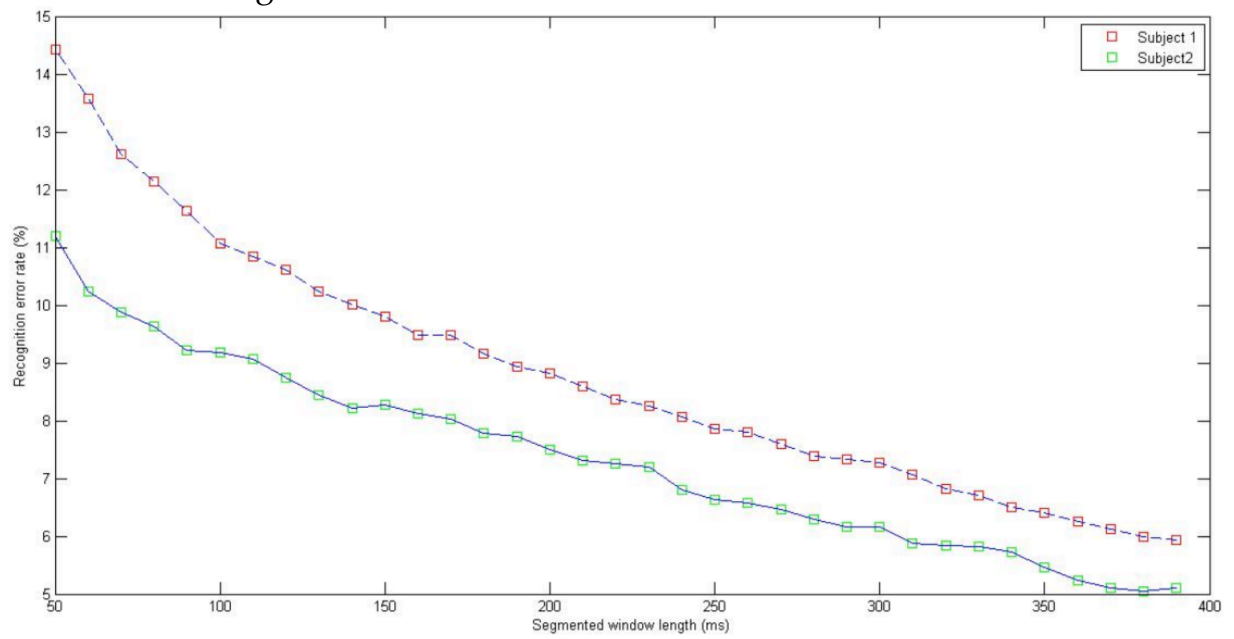

Figure 8. The effect of segmented window length on recognition error rate 


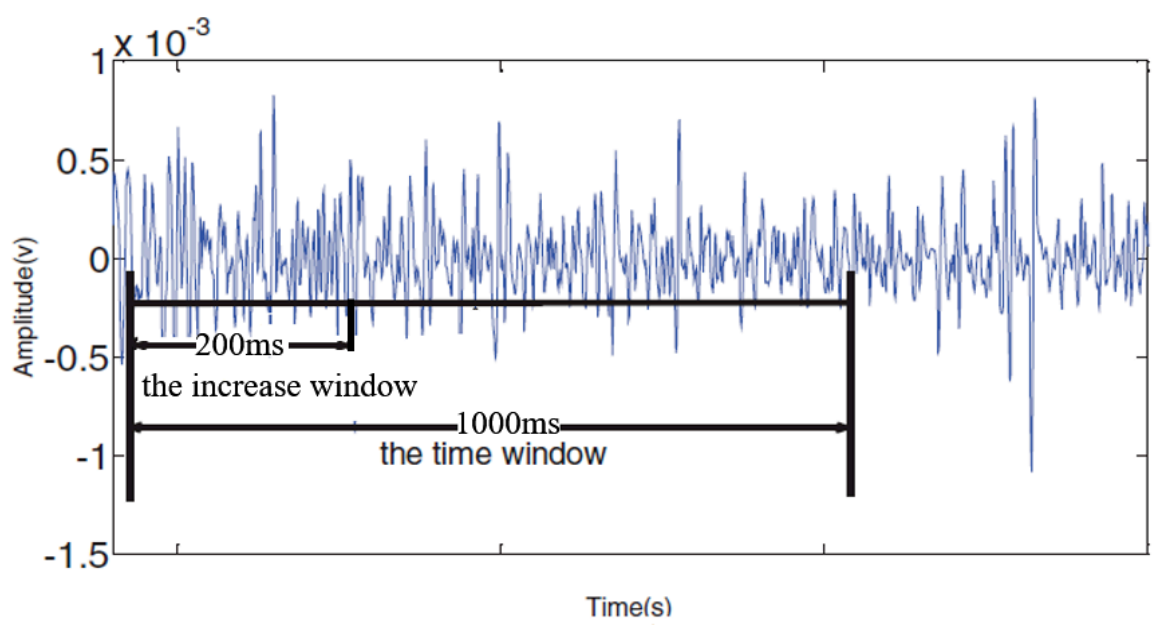

Figure 9. 1000-ms time windows and 200-ms increase window

\subsection{Performance evaluation}

This paper compares this new kind of feature with other four time-domain features to prove the effectiveness of the proposed method. The traditional feature set contains mean absolute value (MAV), waveform length (WL), zero crossing (ZC) and slope sign changes (SSC).

Waveform length (WL) is the cumulative length of the waveform over the time segment [36]. WL is related to the waveform amplitude, frequency and time. Where $\mathrm{N}$ represents the length of the signal and $X_{n}$ represents the EMG signal in a segment. It is given by (9).

$$
W L=\sum_{n=1}^{N-1}\left|X_{n+1}-X_{n}\right|
$$

Zero crossing $(\mathrm{ZC})$ is the number of times that the amplitude value of EMG signal crosses the zero $y$-axis [34]. This feature provides an approximate estimation of frequency domain properties. Where $\mathrm{N}$ represents the length of the signal and $X_{n}$ represents the EMG signal in a segment. It can be formulated as (10).

$$
\begin{gathered}
Z C=\sum_{n=1}^{N-1}\left[\operatorname{sgn}\left(x_{n} \times x_{n+1}\right) \cap\left|x_{n}-x_{n+1}\right| \geq \text { threshold }\right], \\
\operatorname{sgn}(x)=\left\{\begin{array}{l}
1, \text { if } x \geq \text { threshold } \\
0, \text { otherwise }
\end{array}\right.
\end{gathered}
$$

Slope Sign Change (SSC) is similar to ZC and another method to represent the frequency information of EMG signal [35]. The number of changes between positive and negative slope among three consecutive segments are performed with the threshold function for avoiding the interference in EMG signal. Where $\mathrm{N}$ represents the length of the signal and $X_{n}$ represents the EMG signal in a segment. It is defined as (11).

$$
\begin{gathered}
S S C=\sum_{n=2}^{N-1}\left[f\left[\left(x_{n}-x_{n-1}\right) \times\left(x_{n}-x_{n+1}\right)\right]\right] ; \\
f(x)=\left\{\begin{array}{l}
1, \text { if } x \geq \text { threshold } \\
0, \text { otherwise }
\end{array}\right.
\end{gathered}
$$


In terms of classifiers, many researchers proposed and applied lots of methodologies to process and discriminate sEMG signals, including Neural Networks [50,51,52] such as Radial Basis Function Artificial Neural Network [53], statistic approaches such as Hidden Markov Models (HMMs) [54] and Gaussian Mixture Models (GMMs) [50,55], and fuzzy methods $[56,57,58]$. The support vector machine (SVM) is adopted to classify the hand motions classes [59]. SVM is the most widely used and the most comprehensive classifier $[60,62,63]$. It has equivalent performance comparing to other complex classifiers while needs less computing time. This paper uses the LIBSVM software package. LIBSVM is a simple, easy-to-use, fast and effective SVM pattern recognition and regression software package developed by Professor Lin Chih-Jen of Taiwan University. He not only provides compiled executable files that can be used in Windows system, but also provides source code, which is convenient for improvement, modification and application in other operating systems. The software has relatively few adjustments to the parameters involved in SVM, and provides many default parameters, which can solve many problems. It also provides the function of cross validation. When SVM is used in pattern recognition or regression, there is no unified model for SVM method and its parameters, kernel functions and their parameters. Optimal SVM algorithm parameter selection can only rely on experience, experimental comparison, a wide range of search or the use of software packages to provide interactive checking function for optimization.

In this paper, the kernel function of SVM is radial basis function (RBF). RBF is a scalar function along the radial symmetry. It is usually defined as a monotone function of Euclidean distance between $x$ of any point in space and $x c$ of a center. It can be represented as $k(\|x-x c\|)$. The most commonly used radial basis function is the Gauss kernel function $[64,65,66]$. It can be represented as $\mathrm{k}(\|\mathrm{x}-\mathrm{xc}\|)=\exp \left\{-\|\mathrm{x}-\mathrm{xc}\|^{\wedge} 2 /\left(\left(2^{*} \sigma\right)^{\wedge} 2\right)\right\}$. $\mathrm{xc}$ is the center of the kernel function, and $\sigma$ is the width parameter of the function, which controls the radial action range of the function $[67,68,69]$. If $x$ and $x c$ are very close, then the kernel function value is 1 . If the difference between $x$ and $x c$ is very large, then the kernel function value is about 0 . Because this function is similar to the Gauss distribution, it is called the Gauss kernel function, also known as radial basis function (RBF). It can map the original features to infinite dimensions [70,71,72]. Based on the above advantages of RBF, this paper chooses it as the kernel function of SVM.

Half of the trials data are used as training data to construct the classifier and the other half are used as testing data. This papers each feature 12 times [73,74]. On the other hand, this paper tests the average classification errors of specific motion classes among five sEMG features, AMR, MAV, SSC, ZC and WL. This paper uses SVM classifier test five different features (AMR, MAV, SSC, ZC and WL). Their performance is compared to test whether the proposed new feature extraction method can improve the classification accuracy rate of hand motions based on sEMG.

\section{Results}

Figure 10 shows the classification errors over 12 tests by using the five sEMG features. For every test, the AMR obtains the lowest classification error. In addition, the difference of classification error among the tests is stable. This result proves that the training data and testing data are reasonable. At the same time, the results of the experiment are reliable. The average classification errors of AMR, MAV, SSC, ZC and WL across 12 tests are 13\%, 19\%, $26 \%, 24 \%, 22 \%$ respectively as showed in Figure 11 The result shows the classification error of AMR is lower than other traditional features. 


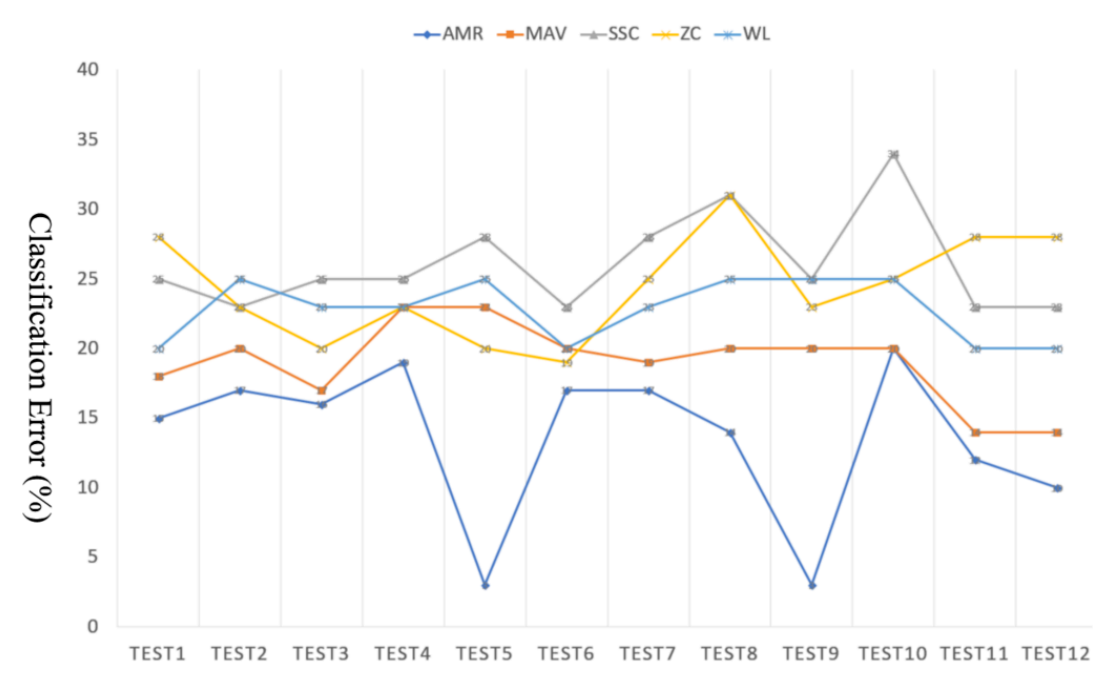

Figure 10. Classification errors over 12 tests by use the five sEMG features

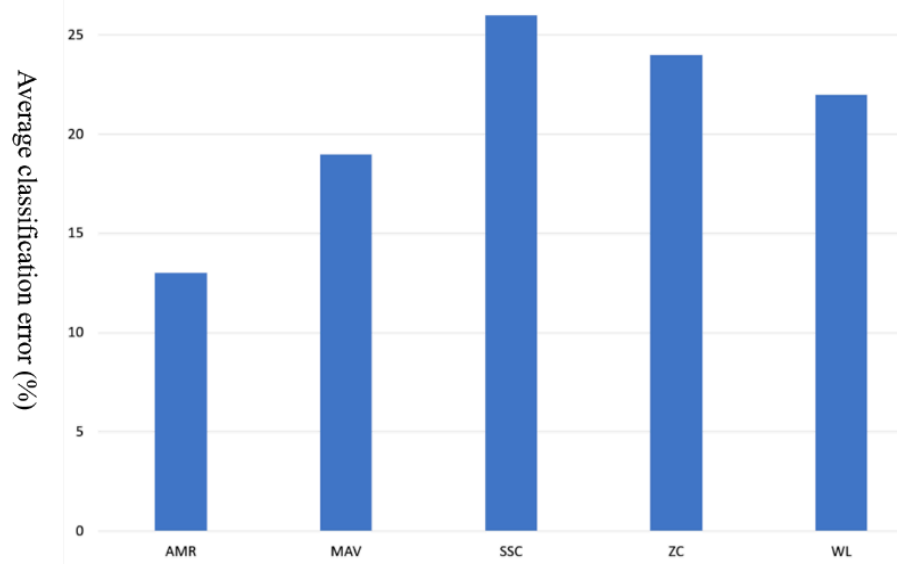

Figure 11. Average classification errors

Figure 12 shows the average classification errors rate of each feature to each hand motion. Comparing with other features, the classification error rate of each motion class of AMR is lower. The feature vector size of AMR is $1 \times 20$. The other time domain feature vector size is $1 \times 90$. AMR can be directly input into the classifier for hand motion classification. The other time domain feature vector need to be normalized firstly. This paper extracts a kind of simple and effective hand motion feature in sEMG signal. This is a nonlinear feature. Compared with the time domain characteristics, this feature has better robustness to various noises.

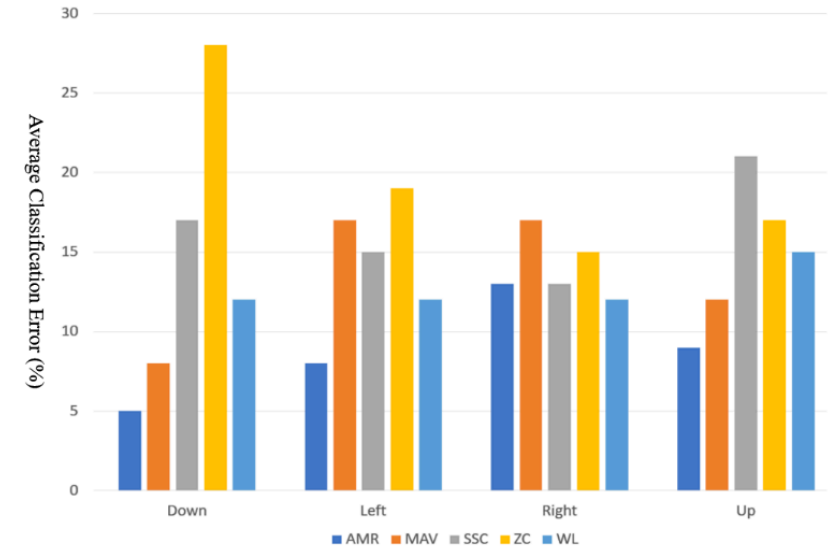

Figure 12. Average classification errors of each feature to each hand motion 


\section{CONCLUSION AND DISCUSSION}

In this study, a new sEMG feature extraction method is proposed. The new feature, active muscle region (AMR), obtains the lowest classification error (average 13\%) when the support vector machine (SVM) is used to compare with other four tradition sEMG feature (average $19 \%, 26 \%, 24 \%$ and $22 \%$ ). While the new feature improves the accuracy of recognition, the new feature vector size is much smaller than other features. This proves that this feature removes redundant information in traditional features. The feature can be directly input into the classifier for hand motion classification. The other time domain feature vector needs to be normalized firstly. Based on the above two reasons, the new features can reduce the computation cost and computation time of pattern recognition methods.

In conclusion, the new sEMG feature extraction method is effective and suitable for the sEMG pattern recognition. The new EMG features are based on the mapping relationship between hand movements and forearm muscle activities. This mapping relationship has been confirmed in medicine. We obtain the active muscle position data from the original EMG signal by the new feature extraction algorithm. The results obtained from this algorithm can well represent hand motions. Future research will use more subjects and more hand motions for data collection and validation. Therefore, more efforts should be used this new feature to establish a real-time hand motion recognition system with machine learning methods. If the high-density electrode is introduced into the experiment, a better classification result is expected. High density electrodes can make fine division of skin surface region. The feature extraction method combined with high density electrode is used to get the active muscle region cloud map. Future, we can use the active muscle region cloud map characterizes hand motions. Finally, we hope to realize the real-time control of prosthetic hand based on EMG by optimizing the algorithm.

Acknowledgments: This work was supported by grants of Natural Science Foundation of China (Grant Nos.51575407, 51575338, 51575412, 61733011), grants of National Natural Science Foundation of China (Grant Nos.51505349, 51575407, 51575338, 51575412, 61733011) and the Grants of National Defense Pre-Research Foundation of Wuhan University of Science and Technology (GF201705). This paper is funded by Wuhan University of Science and Technology graduate students short-term study abroad special funds.

Conflicts of Interest: The authors declare no conflict of interest.

\section{References}

1. Yinfeng, F.; Hettiarachchi, N; Dalin, Z; Honghai, L. Multi-Modal Sensing Techniques for Interfacing Hand Prostheses: A Review. IEEE Sensors Journal 2015, 15, 6065-6076.

2. Castellini, C.; Smagt, P. Surface EMG in advanced hand prosthetics. Biol. Cybern 2009, 100, 35-47.

3. Cipriani, C.; Antfolk, C; Balkenius, C; Rosén, B; Lundborg, G; Carrozza, M; Sebelius, F. A novel concept for a prosthetic hand with a bidirectional interface: A feasibility study. IEEE Trans. Biomed. Eng 2009, 56, 2739-2743.

4. Arieta, A; Yokoi, H; Arai, T; Yu, W. Study on the effects of electrical stimulation on the pattern recognition for an EMG prosthetic application. in Proc. IEEE Int. Conf. Eng. Med. Biol. Soc 2006, 6919-6922.

5. Zhang, D; Chen, X; Li, S; Hu, P; Zhu, X. EMG controlled multifunctional prosthetic hand: Preliminary clinical study and experimental demonstration. in Proc. IEEE Int. Conf. Robot. Autom 2011, 4670-4675.

6. Yang, H; Gongfa, L; Yajie, L; Ying, S; Jianyi K; Guozhang, J; Du, J. Gesture recognition based on an improved local sparse representation classification algorithm. Cluster Computing 2017, DOI: 10.1007/s10586-017-1237-1. 
7. Bei L; Ying, S; Gongfa, L; Jianyi K; Guozhang, J; Du, J; Honghai, L. Gesture recognition based on modified adaptive orthogonal matching pursuit algorithm. Cluster Computing 2017, https://doi.org/10.1007/s10586-017-1231-7.

8. Wei M; Gongfa, L; Guozhang, J; Yinfeng F; Zhaojie J; Honghai L. Optimal grasp planning of multi-fingered robotic hands: a review. Applied and Computational Mathematics 2015, 14(3), 238-247.

9. Gongfa, L; Heng, T; Ying S; Jianyi K; Guozhang J. Hand gesture recognition based on convolution neural network. Cluster Computing 2017, https://doi.org/10.1007/s10586-017-1435-x.

10. Du J, Zujia Z,Gongfa L, Ying S, Jianyi K, Guozhang J, Hegen X, Bo T, Shuang X, Hui Y, Honghai L, Zhaojie J. Gesture recognition based on binocular vision. Cluster Computing 2018, https://doi.org/10.1007/s10586-018-1844-5.

11. Ying S, Cuiqiao L, Gongfa L, Guozhang J, Du J, Honghai L, Zhigao Z, Wanneng S. Gesture Recognition Based on Kinect and sEMG Signal Fusion. Mobile Networks and Applications 2018, https://doi.org/10.1007/s11036-018-1008-0.

12. Weiliang D; Gongfa, L; Guozhang, J; Yinfeng F; Zhaojie J; Honghai L. Intelligent computation in grasping control of dexterous robot hand. Journal of Computational \& Theoretical Nanoscience 2015, 12(12), 6096-6099.

13. Zhe L; Gongfa, L; Guozhang, J; Yinfeng F; Zhaojie J; Honghai L. Intelligent Computation of grasping and manipulation for multi-fingered robotic hands. Journal of Computational \& Theoretical Nanoscience 2015, 12(12), 6192-6197.

14. Scott, R. Myoelectric control of prostheses. Archives of physical medicine and rehabilitation 1966, $47,174$.

15. Zhang, F.; Li, P; Hou, Z. sEMG-based continuous estimation of joint angles of human legs by using BP neural network. Neurocomputing 2012, 78, 139-148.

16. Li, Z.; Wang, B; Sun, F; Yang, C. sEMG-based joint force control for an upper-limb power-assist exoskeleton robot. IEEE J Biomed Health Inform 2014, 18, 1043-1050.

17. Lee, J.; Kim, M; Kim, K. A Robust Control Method of Multi-DOF Power-Assistant Robots for Unknown External Perturbation using sEMG Signals. Ieee/Rsj International Conference on Intelligent Robots and Systems (Iros) 2015, 1045-1051.

18. Jianda, H.; Qichuan, D; Anbin, X; Xingang, Z. A State-Space EMG Model for the Estimation of Continuous Joint Movements. IEEE Transactions on Industrial Electronics 2015, 62, 4267-4275.

19. Daley, H.; Englehart, K; Hargrove, L; Kuruganti, U. High density electromyography data of normally limbed and transradial amputee subjects for multifunction prosthetic control. Electromyogr. Kines 2015, 22, 478-484.

20. Li, G.; Schultz, A; Kuiken, T. Quantifying pattern recognition based myoelectric control of multifunctional transradial prostheses. Neural Systems and Rehabilitation Engineering 2010, 18, 185-192.

21. Orosco, E.; Lopez, N; Sciascio, F. Bispectrum-based features classification for myoelectric control. Biomed. Signal Process. Control 2013, 8, 153-168.

22. Angkoon, P.; Franck, Q; Sylvie, C; Christine, S; Frank, T; Yann, L. EMG feature evaluation for improving myoelectric pattern recognition robustness. Expert Systems with Applications 2013, 40, 4832-4840.

23. Hudgins, B.; Parker, P; Scott, R. A new strategy for multifunction myoelectric control. Biomedical Engineering, IEEE Transactions on 1993, 40, 82-94.

24. Englehart, K.; Hudgins, B; Parker, P. A wavelet-based continuous classification scheme for multifunction myoelectric control. Biomedical Engineering, IEEE Transactions on 2001, 48, 302-311.

25. Chen, X.; Zhu, X.; Zhang, D. A discriminant bispectrum feature for surface electromyogram signal classification. Medical engineering \& physics 2010, 32, 126-135.

26. Graupe, D.; Salahi, J.; Zhang D. Stochastic analysis of myoelectric temporal signatures for multifunctional single-site activation of prostheses and orthoses. Journal of biomedical engineering $1985,7,18-29$.

27. Yinfeng, F.; Honghai, L.; Gongfa, Li.; Xiangyang, Z. A Multichannel Surface EMG System for Hand Motion Recognition. International Journal of Humanoid Robotics 2015, 12, 381-509. 
28. Young, A.; Hargrove L.; Kuiken, T. Improving myoelectric pattern recognition robustness to electrode shift by changing interelectrode distance and electrode configuration. IEEE Transactions on Biomedical Engineering 2012, 59, 645-652.

29. Oskoei, M.; Hu, H. Support vector machine-based classification scheme for myoelectric control applied to upper limb. IEEE Transactions on Biomedical Engineering 2008, 55, 1956 -1965.

30. Zhaojie, J.; Gaoxiang, O.; Korsak, W.; Honghai, L. Surface EMG Based Hand Manipulation Identification Via Nonlinear Feature Extraction and Classification. IEEE Sensors Journal 2013, 13, 3302-3311.

31. Poosapadi, A.; Kumar, D.; Computation of fractal features based on the fractal analysis of surface electromyogram to estimate force of contraction of different muscles. Comput Methods Biomech Biomed Engin, 2014, 17, 210-216.

32. Ancillao, A.; Galli, M.; Rigoldi, C. Albertini, G. Linear correlation between fractal dimension of surface EMG signal from Rectus Femoris and height of vertical jump. Chaos Solitons \& Fractals 2014, 66, 120-126.

33. Gongfa, L.; Yuesheng, G.; Jianyi, K.; Guozhang, J.; Liangxi, X.; Zehao, W.; Zhen, L.; Yuan, H.; Po, G . Intelligent control of air compressor production process. Applied Mathematics \& Information Sciences, 2013, 7(3), 1051-1058.

34. Xinpu, C.; Xiangyang, Z.; Dingguo, Z. A discriminant bispectrum feature for surface electromyogram signal classification. Medical Engineering \& Physics 2010, 22, 126-135.

35. Subasi, A. Classification of EMG signals using combined features and soft computing techniques. Applied Soft Computing Journal 2012, 12, 2188-2198.

36. Lukai, L.; Pu, L.; Edward, A. Electromyogram whitening for improved classification accuracy in upper limb prosthesis control. IEEE Trans Neural Syst Rehabil Eng 2013, 21, 767-774.

37. Yang, L.; Yantao, T.; Wanzhong, C. Modeling and Classifying of sEMG Based on FFT Blind Identification. Acta Automatica Sinica 2012, 38(1), 128-134.

38. Gongfa, L.; Peixin, Q.; Jianyi, K.; Guozhang, J.; Liangxi, X.; Po, G.; Zehao, W.; Yuan, He. Coke oven intelligent integrated control system. Applied Mathematics \& Information Sciences, 2013, 7(3), 1043-1050.

39. Gang, W.; Zhizhong, W.; Weiting, C.; Jun, Z. Classification of surface EMG signals using optimal wavelet packet method based on Davies- Bouldin criterion. Med Biol Eng Comput 2006, 44(10), 865-72.

40. Wenjun, C.; Gongfa, L.; Jianyi, K.; Ying, S.; Guozhang, J.; Honghai, L. Thermal Mechanical Stress Analysis of Ladle Lining with Integral Brick Joint. Archives of Metallurgy and Materials. 2018, 63, 659-666.

41. Andrews, A.; Morin, E.; Mclean, L. Optimal electrode configurations for finger movement classification using EMG. Engineering in Medicine and Biology Society 2009, 2987-2990.

42. Hargrove, L; Englehart, K.; Hudgins, B. A training strategy to reduce classification degradation due to electrode displacements in pattern recognition based myoelectric control. Biomedical Signal Processing and Control 2008, 3(2), 175-180.

43. Gongfa, L.; Peixin, Q.; Jianyi, K.; Guozhang, J.; Liangxi, X.; Zehao, W.; Po, Gao.; Yuan, He. Influence of working lining parameters on temperature and stress field of ladle. Applied Mathematics \& Information Sciences, 2013, 7(2), 439-448.

44. Hargrove, L.; Englehart, K.; Hudgins, B. The effect of electrode displacements on pattern recognition based myoelectric control. Engineering in Medicine and Biology Society 2006, 2203-2206.

45. Mojtaba, M.; Farbod, R.; ShMahdi, A. Elimination of power line noise from EMG signals using an efficient adaptive Laguerre filter. IEEE International Conference on Signals and Electronic Systems 2010, 49-52.

46. Hegen, X.; Huali, F.; Gongfa, L.; Guozhang, J. Research on steady-state simulation in dynamic job shop scheduling problem. Advances in Mechanical Engineering, 2015, 7(9), 1-11.

47. Guanglin, L.; Yanjuan G; Dandan T; Ping Z. Performance of electromyography recorded using textile electrodes in classifying arm movements. Conf. Proc. IEEE Eng. Med. Biol. Soc, 2011, 4243-4246. 
48. Carlo, L.; Donald Gilmore, L; Mikhail, K; Serge, B. Filtering the surface EMG signal: Movement artifact and baseline noise contamination. Journal of Biomechanics 2010, 43, 1573-1579.

49. Bernard, H.; Philip, P; Robert, S. A new strategy for multifunction myoelectric control. IEEE Trans. Biomed. Eng 1993, 40, 82-84.

50. Dapeng, Y.; Jingdong, Z; Jiang, L; Hong L. Dynamic hand motion recognition based on transient and steady-state EMG signals. International Journal of Humanoid Robotics 2012, 9, 1250-1256.

51. Shenkai, C.; Mingtung, W; Chunhao, H; Jiahroung, W; Lanyuen, G; Wenlan, W. The analysis of upper limb movement and EMG activation during the snatch under various loading conditions. Journal of Mechanics in Medicine \& Biology 2013, 13, 1350-1357.

52. Md, A.; Muhammad, I; Othman K. Electromygraphy (EMG) Signal based Hand Gesture Recognition using Artificial Neural Network (ANN). 4th International Conference on Mechatronics. Malaysia 2011,4,1-6.

53. Momen, K.; Krishnan, S.; Chau, T. Real-time classification of forearm electromyographic signals corresponding to user-selected intentional movements for multifunction prosthesis control. IEEE Transactions on Neural Systems \& Rehabilitation Engineering 2007, 15, 535-542.

54. Englehart, K.; Hudgins, B. A robust real-time control scheme for multifunction myoelectric control. IEEE Trans on Biomedical Engineering 2003, 50, 848-854.

55. Gongfa, L.; Ze, L.; Guozhang, J.; Hegen, Xiong.; Honghai, Liu. Numerical simulation of the influence factors for rotary kiln in temperature field and stress field and the structure optimization . Advances in Mechanical Engineering, 2015, 7(6), 1-15.

56. Veternik, M.; Simera, M.; Jakus, J.; Poliacek, I. Integration of simulated multipotential signals: the role of integration window width and of the number of spikes. Neurobiology of Respiration. Springer Netherlands 2013, 788, 265-272.

57. Chen, X.; Zhu, X.; Zhang, D. A discriminant bispectrum feature for surface electromyogram signal classification. Medical Engineering \& Physics 2010, 32, 126-35.

58. Bu, N.; Hamamoto, T.; Fukuda, O.; Tsuji. FPGA implementation of a probabilistic neural network. IEICE Transactions on Information \& Systems 2005, 88, 390-397.

59. Gongfa, L.; Jianyi, K.; Guozhang, J.; Liangxi, X.; Zhigang, J.; Gang, Z.; Air-fuel ratio intelligent control in coke oven combustion process. Information-An International Interdisciplinary Journal, 2012, 15, 4487-4494.

60. Ahsan, M.; Ibrahimy, M.; Khalifa, O. EMG signal classification for human computer interaction: A review. European Journal of Scientific Research 2009, 33, 480-501.

61. Mobasser, F.; Hashtrudi-Zaad, K. A method for online estimation of human arm dynamics. Engineering in Medicine and Biology Society 2006, 2412-2416.

62. Gongfa, L.; Jia, L.; Guozhang, J.; Honghai, L. Numerical simulation of temperature field and thermal stress field in the new type of ladle with the nanometer adiabatic material. Advances in Mechanical Engineering, 2015, 7(4),1-13.

63. Chan, A.; Englehart K. Continuous myoelectric control for powered prostheses using hidden Markov models. IEEE Trans. Biomed. Eng 2005, 52, 121-124.

64. Huang, Y.; Englehart, K.; Hudgins, B.; Chan, A. A Gaussian mixture model based classification scheme for myoelectric control of powered upper limb prostheses. IEEE Trans. Biomed. Eng 2005, 52, 1801-1811.

65. Khezri, M.; Jahed, M. Real-time intelligent pattern recognition algorithm for surface EMG signals. Biomedical Engineering Online 2007, 6, 45.

66. Disi C.; Gongfa L. An interactive image segmentation method in hand gesture recognition. Sensors $2017,17(2), 253$.

67. Yajie L.; Ying S.; Gongfa L.; Jianyi K. Simultaneous calibration: a joint optimization approach for multiple kinect and external cameras. Sensors 2017, 17(7): 1491.

68. Disi, C.; Gongfa, L.; Ying S.; Guozhang J.; Jianyi K.; Jiahan L.; Honghai L.; Fusion hand gesture segmentation and extraction based on CMOS sensor and 3D sensor. International Journal of Wireless and Mobile Computing, 2017, 12(3): 305-312.

69. Ying, S.; Jiabing, H.; Practice Teaching of Mechanical Design Based on Computer Media Simulation. The Journal of Supercomputing, 2018, https://doi.org/10.1007/s11227-018-2255-3. 
70. Qian, Y.; Gongfa, Li.; Jiangguo Z.; Research on the method of step feature extraction for EOD robot based on 2d laser radar. Discrete and continuous dynamical system-series, 2015, 8(6): 1415-1421.

71. Gongfa, L.; Leilei, Z.; Ying, S.; Jianyi K. Internet of Things sensors and haptic feedback for sEMG based hands . Multimedia Tools and Applications,2018, https://doi.org/10.1007/s11042-018-6293-x

72. Yang, H.; Gongfa, L.; Yaping, Z.; Ying, S.; Guozhang, J. Numerical Simulation-based Optimization of Contact Stress Distribution and Lubrication Conditions in the Straight Worm Drive. Strength of Materials,2018,50(1):157-165.

73. Gongfa, L.; Wei, M.; Guozhang, J.; Yinfeng, F.; Zhaojie, J.; Honghai, L. Intelligent control model and its simulation of flue temperature in coke oven. Discrete and Continuous Dynamical Systems Series S (DCDS-S), 2015, 8(6), 1223-1237.

74. Hegen, X.; Huali, F.; Guozhang, J.; Gongfa, Li. A simulation -based study of dispatching rules in a dynamic job shop scheduling problem with batch release and extended technical precedence constraints, European Journal of Operational Research, 2017, 257(1),13-24. 